\title{
Cenário epidemiológico dos casos notificados do HIV/Aids em Alagoas no período de
}

\section{9 a 2018}

Epidemiological scenario of notified cases of HIV/AIDS in Alagoas from 2009 to 2018

Escenario epidemiológico de casos notificados de HIV/SIDA en Alagoas de 2009 a 2018

Recebido: 08/04/2021 | Revisado: 16/04/2021 | Aceito: 16/04/2021 | Publicado: 02/05/2021

Daniella Marques dos Santos Mariano

ORCID: https://orcid.org/0000-0001-8977-4149 Universidade Federal de Alagoas, Brasil

E-mail: quirimarques@ hotmail.com

Keila Cristina do Nascimento Oliveira

ORCID: https://orcid.org/0000-0003-0167-5889 Universidade Federal de Alagoas, Brasil

E-mail: keila.oliveira@eenf.ufal.br

Diego Pereira Gonçalo da Hora

ORCID: https://orcid.org/0000-0003-2123-4976 Secretaria de Estado da Saúde de Alagoas, Brasil E-mail: diegopereira.84@hotmail.com

Viviane Vanessa Rodrigues da Silva Santana Lima ORCID: https://orcid.org/0000-0001-6903-1444 Universidade Federal de Alagoas, Brasil E-mail: viviane.santana@eenf.ufal.br

Alysson Mariano Alves

ORCID: https://orcid.org/0000-0001-8809-8791 Universidade Federal de Alagoas, Brasil

E-mail: alyssonmarianoalves@ hotmail.com

Thainá da Silva Cabral

ORCID: https://orcid.org/0000-0002-6972-2904 Universidade Federal de Alagoas, Brasil E-mail: thainacabral.cabral@gmail.com

Marcela Cristina dos Santos Barros

ORCID: https://orcid.org/0000-0001-7863-145X Universidade Federal de Alagoas, Brasil E-mail:mh0673@gmail.com Igor Michel Ramos dos Santos ORCID: https://orcid.org/0000-0001-6557-3369 Universidade Federal de Alagoas, Brasil E-mail: igor.santos@esenfar.ufal.br

Vanessa Vieira de Souza Oliveira ORCID: https://orcid.org/0000-0001-7341-0615 Universidade Federal de Alagoas, Brasil E-mail: vanessavso25@gmail.com

Roberto da Silva Bezerra

ORCID: https://orcid.org/0000-0001-7625-075X Universidade Federal de Alagoas, Brasil E-mail: betobezerra21@gmail.com

\section{Resumo}

Objetivo: Realizar um levantamento acerca do cenário epidemiológico de HIV/Aids em Alagoas. Método: Trata-se de estudo epidemiológico descritivo com abordagem quantitativa acerca do cenário do HIV e da Aids no estado de Alagoas no período de 2009 a 2018, utilizando dados secundários do SINAN por meio da Ficha de Notificação/Investigação Aids (pacientes com 13 anos ou mais) disponibilizados pela Secretaria de Saúde do Estado de Alagoas (SESAU), tabulados em planilha eletrônica (Microsoft Excel®) e analisados estatisticamente através do software Statistical Package for the Social Sciences (SPSS) 22.0. As variáveis constituintes desse estudo são: dados sociodemográficos, vias de transmissão do HIV, critérios de definição de casos de HIV/Aids e evolução dos casos notificados. Resultados: Foram notificados 7.447 casos de HIV e Aids no período observado, sendo 62,9\% de indivíduos do sexo masculino e 37,1\% do sexo feminino, apresentando média de idade de 35 anos, com $71,5 \%$ de pessoas da raça/cor parda e $44,5 \%$ de pessoas analfabetas ou que possuem até o ensino fundamental completo. A categoria de exposição mais frequente foi a heterossexual, consideravelmente maior entre as mulheres. Dos 102 municípios de Alagoas, 96 deles (94,12\%) apresentaram casos de HIV e Aids. O estudo revela ainda que há aumento 
anual do número de novos casos notificados de HIV e diminuição do número de óbitos por Aids. Conclusão: O perfil epidemiológico do HIV/Aids em Alagoas é permeado pelos processos de vulnerabilidade social e de gênero, com o cenário apresentando tendências de interiorização, juvenização, feminização e pauperização ao longo da série histórica analisada.

Palavras-chave: Perfil epidemiológico; HIV/Aids; Alagoas.

\begin{abstract}
Objective: Conduct a survey about the epidemiological scenario of HIV/Aids in Alagoas. Method: This is a descriptive epidemiological study with a quantitative approach on the scenario of HIV and Aids in the state of Alagoas from 2009 to 2018, using secondary data from SINAN through the Aids Notification / Investigation Form (patients aged 13 or over) available by the Health Department of the State of Alagoas (SESAU), tabulated in electronic spreadsheet (Microsoft Excel®) and analyzed statistically using the Statistical Package for the Social Sciences (SPSS) 22.0. The constituent variables of this study are sociodemographic data, HIV transmission routes, criteria for defining cases of HIV/Aids and evolution of reported cases. Results: Were reported 7,447 cases of HIV and Aids in the observed period, with $62.9 \%$ of males and $37.1 \%$ of females, with an average age of 35 years, with $71.5 \%$ of people of race/color brown and $44.5 \%$ of people who are illiterate or who have completed elementary school only. The most frequent category of exposure was heterosexual, considerably higher among women. Of the 102 municipalities belonging to the state of Alagoas, 96 of them (94.12\%) presented cases of HIV and Aids. The study also reveals that there is an annual increase in the number of new diagnosed HIV cases and a decrease in the number of deaths from Aids. Conclusion: The epidemiological profile of HIV/Aids in Alagoas is permeated by the processes of social and gender vulnerability, with the scenario showing trends of interiorization, youth, feminization and impoverishment throughout the analyzed historical serie.
\end{abstract}

Keywords: Epidemiological profile; HIV/Aids; Alagoas.

\begin{abstract}
Resumen
Objetivo: Realizar una encuesta sobre el escenario epidemiológico del HIV/SIDA en Alagoas. Método: Se trata de un estudio epidemiológico descriptivo con enfoque cuantitativo sobre el escenario de HIV y SIDA en el estado de Alagoas de 2009 a 2018, utilizando datos secundarios del SINAN a través del Formulario de Notificación/Investigación de SIDA (pacientes de 13 años o más) disponible por la Secretaría de Salud del Estado de Alagoas (SESAU), tabulado en hoja electrónica (Microsoft Excel®) y analizado estadísticamente mediante el software Statistical Package for the Social Sciences (SPSS) 22.0. Las variables constitutivas de este estudio son: datos sociodemográficos, vías de transmisión del HIV, criterios para la definición de casos de HIV/SIDA y evolución de los casos notificados. Resultados: Se reportaron 7.447 casos de HIV y SIDA en el período observado, con 62,9\% de hombres y $37,1 \%$ de mujeres, con una edad promedio de 35 años, con 71,5\% de personas de raza/color marrón y $44,5 \%$ de personas que son analfabetos o que han completado la escuela primaria. La categoría de exposición más frecuente fue la heterosexual, considerablemente más alta entre las mujeres. De los 102 municipios de Alagoas, 96 $(94,12 \%)$ tenían casos de HIV y SIDA. El estudio también revela que hay un aumento anual en el número de nuevos casos notificados de HIV y una disminución en el número de muertes por SIDA. Conclusión: El perfil epidemiológico del HIV/SIDA en Alagoas está permeado por los procesos de vulnerabilidad social y de género, con el escenario mostrando tendencias de interiorización, juventud, feminización y empobrecimiento a lo largo de la serie histórica analizada.
\end{abstract}

Palabras clave: Perfil epidemiológico; HIV/SIDA; Alagoas.

\title{
1. Introdução
}

A síndrome da imunodeficiência adquirida (Aids) é um problema de saúde pública global e que desde o início de sua epidemia na década de 1980 vem vitimando milhões de pessoas no mundo, em especial a população que vive em situação de vulnerabilidade social, econômica e demográfica (Fiocruz, entre 2007 e 2020; Unaids, 2012).

Atualmente, organizações de saúde do mundo todo reconhecem a necessidade de frear a disseminação do vírus causador da Aids. Para isso, vários países do mundo vêm investindo anualmente em políticas públicas de combate à propagação do vírus e tratamento dos doentes, uma vez que não existe cura para tal infecção (Unaids, 2012).

No Brasil, a fim de se conhecer o panorama epidemiológico da Aids e de outras doenças infecciosas e agravos, o país dispõe do sistema de vigilância epidemiológica que tem por objetivo sugerir, planejar e implantar medidas de intervenção para o processo de saúde-doença. Ademais, a vigilância epidemiológica conta com o sistema de informação de saúde que permite a análise da situação de saúde desde o nível local até o nacional (Medronho, 2009). 
Dentre os sistemas de informação de saúde o presente estudo apresenta como base os dados provenientes do Sistema de Informação de Agravos de Notificação (SINAN) que desde 1993 vem sendo alimentado por estados e municípios acerca das doenças e agravos que constam na lista de notificação compulsória do país, colaborando assim para o conhecimento da realidade epidemiológica de determinada área geográfica (Medronho, 2009; Sinan, entre 2017 e 2020).

Nos anos anteriores a 2014 não havia notificação efetiva de casos de HIV no Brasil, porque desde o início da notificação compulsória na década de 1980 foram notificados somente os casos de indivíduos que já tinham desenvolvido a Aids, gestantes e puérperas com HIV e as crianças expostas ao vírus (Brasil, 2019a). Deste modo, a provável causa para o aumento no número de casos notificados de HIV em 2014 pode ser justificada pelo fato de que somente a partir de 2014 a notificação compulsória dos casos de HIV passou a ser regulamentada pela portaria n 1.271/2014 (Brasil, entre 2016 e 2020).

Entre os anos de 2007 a 2018 o SINAN notificou no Brasil 300.496 casos de infecção pelo HIV, revelando um crescimento anual em todas as regiões brasileiras, em especial no Nordeste onde se evidenciou um aumento preocupante, com os casos notificados saltando de 8,7\% em 2007 para 37,7\% em 2018. Em Alagoas, desde o início da epidemia de Aids em 1980 até o ano de 2019, foram notificados no SINAN 8.168 casos de HIV e Aids, e registrados 2.161 óbitos decorrentes da Aids (Brasil, 2019a).

Objetivando traçar um perfil da distribuição dos casos notificados de HIV e Aids no estado de Alagoas ao longo de uma série histórica, o presente estudo apresenta seu problema de pesquisa sob a forma do seguinte questionamento: Qual o cenário epidemiológico da ocorrência de HIV/Aids em Alagoas no período de 2009 a 2018 ?

Para que o problema de pesquisa possa ser respondido de maneira eficiente constituem o rol de objetivos específicos do estudo ações como a verificação dos dados sociodemográficos dos casos notificados, a identificação das vias de transmissão do HIV, além da análise de critérios de definição e evolução dos casos notificados.

A justificativa deste trabalho se dá pela possibilidade de um maior aprofundamento acerca da realidade dos casos notificados de HIV e de Aids existentes em Alagoas, considerando em suas bases analíticas a identificação de tendências, o apontamento do perfil sociodemográficos e a maneira como se processa a evolução de casos do conjunto de dados.

O que torna o estudo relevante é a sua condução através de um recorte temporal inédito em meio ao cenário acadêmico no que se refere ao contexto epidemiológico do HIV e da Aids existente no estado de Alagoas, debruçando sua avaliação em um arcabouço documental compilado entre os anos de 2009 a 2018.

\section{Metodologia}

Trata-se de um estudo epidemiológico descritivo realizado no estado de Alagoas com abordagem quantitativa, com a utilização dos dados secundários da Secretaria de Saúde do Estado de Alagoas (SESAU) acerca do cenário do HIV e da Aids no período entre 2009 a 2018 no estado de Alagoas.

Vale enfatizar que no estudo descritivo o pesquisador não interfere sobre os dados coletados, portanto os dados coletados são "observados, registrados, analisados, classificados e interpretados” (Prodanov \& Freitas, 2013, p. 52).

Ainda de acordo com Prodanov e Freitas (2013), atrelado ao estudo descritivo se tem a pesquisa quantitativa que utiliza recursos e técnicas estatísticas para quantificar os dados pesquisados e auxiliar na elaboração dos resultados.

Os dados são de pessoas com 13 (treze) anos ou mais notificados com HIV/Aids no SINAN através da Ficha de Notificação/Investigação Aids, durante o período de 2009 a 2018 no estado de Alagoas, totalizando 7.463 pessoas notificadas.

A amostra descartou um total de 16 (dezesseis) pessoas notificadas dentro da população supracitada, tomando como base o critério de exclusão que será descrito na sequência. Desta feita, a amostragem da pesquisa apresentou um total de 7.447 pessoas que atendiam aos critérios propostos pelo presente estudo. 
O critério de exclusão definido neste estudo foi composto por indivíduos residentes em outros estados da federação, mas que foram notificados no estado de Alagoas. Vale destacar que pacientes menores de 13 anos, crianças expostas ao HIV, além de gestantes, parturientes e puérperas HIV positivas apresentam seus casos notificados em sistemas específicos, não fazendo parte, portanto, do banco de dados analisado por este trabalho.

A coleta dos dados se deu por meio de informações constantes no SINAN, disponibilizados pela SESAU. Os dados foram solicitados através de ofício protocolado junto à SESAU e disponibilizados pela secretaria via e-mail da pesquisadora no formato de planilha eletrônica (Microsoft Excel®). As variáveis extraídas do SINAN tomaram como base dados sociodemográficos, vias de transmissão do HIV, critérios de definição de casos de HIV/Aids e evolução dos casos notificados.

Os dados visualizados no banco de dados da SESAU encontravam-se dispostos através de planilha eletrônica (Microsoft Excel®) contendo dados que foram traduzidos a partir do dicionário de dados SINAN NET versão 5.0 e, posteriormente, analisados estatisticamente através do software Statistical Package for the Social Sciences (SPSS) 22.0, que possibilitou trabalhar com distribuições de frequência, tabulações simples e cruzadas, além de taxa de incidência e média.

Os resultados do presente estudo foram apresentados em formato de tabelas e gráficos, e a discussão foi fundamentada na literatura disponível acerca do tema.

\section{Resultados e Discussão}

O estado de Alagoas fica localizado na região nordeste do Brasil, sua capital é Maceió e tem como limites os estados de Pernambuco, Sergipe e Bahia, ocupando uma área de $27848.140 \mathrm{~km}^{2}$ e possuindo cento e dois (102) municípios. Segundo censo do Instituto Brasileiro de Geografia e Estatística (IBGE, 2019) até 2010 o estado tinha uma população de 3.120 .494 habitantes e em 2019 a população estimada foi de 3.337 .357 pessoas.

Entre os anos de 2014 a 2018 o estado de Alagoas ocupou a 13 posição no ranking nacional de detecção de Aids na população geral dentre os estados brasileiros, além da quarta posição da região nordeste dentre os nove (9) estados que a compõem. Além disso, a capital Maceió ocupou a $12^{\mathrm{a}}$ posição entre as capitais brasileiras de detecção de Aids na população geral (Brasil, 2019a).

O estudo aponta que no estado de Alagoas foram notificados 7.447 casos de HIV e Aids durante os anos de 2009 a 2018, com uma média de 744,7 casos/ano. Os resultados demonstram que houve um crescimento no número de casos de HIV/Aids notificados em Alagoas durante o período estudado, partindo de 390 (5,2\%) casos em 2009 para 1.325 (17,8\%) casos em 2018, sendo o ano de 2018 o ano com maior com número de diagnósticos. Esse evento possui reflexo direto no crescimento da taxa de incidência apresentada no estado, tendo em vista que ao longo dos anos o estado não apresentou mudanças significativas em sua população (Sidra, entre 2017 e 2020).

Em uma análise acerca da taxa de incidência de casos de Aids, quando se confrontam os dados relativos a Alagoas com dados da região nordeste constata-se um padrão condizente. Todavia, quando se relacionam as taxas de incidência de Alagoas com as taxas nacionais observa-se um padrão divergente. $O$ estado alagoano aumentou a sua taxa de incidência de casos de Aids durante o período analisado, saindo de uma taxa de 11,2 casos de Aids notificados por 100.000 habitantes em 2009 para uma taxa de 13,3 em 2018, o que demonstra uma perspectiva de crescimento da taxa de incidência em âmbito estadual.

Em âmbito regional percebe-se que o Nordeste também apresenta uma tendência de crescimento na incidência de casos de Aids, a exemplo do ano de 2009 onde se registrou uma taxa de 14,1 casos por 100.000 habitantes, subindo para 15,8 no ano de 2018. Portanto, Alagoas segue a tendência de crescimento da taxa de incidência identificada na região nordeste (Brasil, 2019a). 
Os índices nacionais de taxa de incidência, por sua vez, apresentam tendência decrescente ao longo dos anos que compõem o presente estudo, reduzindo de 21,3 casos de Aids notificados por 100.000 habitantes em 2009 para uma taxa de 17,8 em 2018 (Brasil, 2019b).

É importante salientar que o país apresenta uma epidemia multifacetada de disseminação de HIV e Aids, uma vez que coexistem perfis epidemiológicos diferentes em meio às regiões brasileiras, incorrendo em diferentes patamares para cada região de acordo com suas especificidades (Mombelli et al., 2015).

Tomando-se como base toda a série temporal analisada, a capital alagoana apresenta mais da metade dos casos notificados de HIV e Aids do estado (57,3\% dos casos). Em análise comparativa com outra capital nordestina, identificou-se em estudo realizado no Ceará que no ano de 2012 53,7\% dos casos notificados de Aids residiam na capital Fortaleza, observando-se percentuais semelhantes aos encontrados em Maceió (Pedrosa et al., 2015).

Além dos altos índices de casos notificados na capital é notório que existe também um processo de interiorização da doença entre os demais municípios de Alagoas. Entende-se por interiorização a disseminação da epidemia da Aids para os municípios mais longínquos da capital do estado e da região metropolitana, afetando desta forma as pessoas que vivem nessas regiões mais desassistidas em políticas públicas de saúde. Vale enfatizar que esse fenômeno, atualmente, segue a tendência do Brasil que a partir do final da década de oitenta difundiu geograficamente a doença a começar das capitais em direção aos municípios interioranos do país (Brito et al., 2001; Reis et al., 2008).

Ainda de acordo com Brito et. al (2001) apesar de fazer parte de grandes centros urbanos, em meados de 2000 a epidemia da Aids já havia se dispersado para o interior dos estados brasileiros e já atingia naquela época 59\% dos 5.507 municípios brasileiros.

A tendência de interiorização da epidemia citada acima pode ser vista em Alagoas no período estudado. Dos 102 municípios pertencentes ao estado de Alagoas, 96 deles (94,1\%) apresentaram casos notificados de HIV e Aids. Além de Maceió com 4.265 casos (57,3\%) e Arapiraca com 508 casos (6,8\%), outros 7 (sete) municípios possuem mais de cem (100) casos de HIV e Aids notificados no período em análise, a saber: Matriz do Camaragibe com 104 casos (1,4\%); Murici com 115 casos (1,5\%); São Miguel dos Campos com 116 casos (1,6\%); Palmeira dos Índios com 119 (1,6\%); União dos Palmares com 125 casos (1,7\%); Marechal Deodoro com 128 casos (1,7\%) e Rio Largo com 143 casos (1,9\%).

Além do processo de interiorização, o presente estudo apresenta tendências de feminização, juvenização e pauperização em meio aos casos de infecção pelo HIV e de Aids notificados em Alagoas, evidenciando uma realidade condizente com os estudos elaborados por Pedrosa et al. (2015) no estado do Ceará.

Quando a abordagem toma como base o gênero revela-se uma tendência de crescimento no percentual de casos dentro do sexo feminino no estado de Alagoas, configurando-se assim um processo de feminização. Na literatura científica o processo de feminização da Aids é representado não somente pela diferença de gênero, mas também pelas diferenças biológicas e sociais. Além disso, essas mulheres encontram-se no contexto social de vulnerabilidade quando comparadas ao sexo oposto, e essas diferenças refletem diretamente no processo saúde-doença (Paiva, 1999; Botti et al., 2009).

Na série histórica, dos 10 (dez) anos analisados, observam-se percentuais de frequência relativa maiores de mulheres com relação aos homens em 6 ocasiões (2009, 2011, 2012, 2013, 2014 e 2015), ilustrando com o passar dos anos uma maior presença das mulheres no rol de pessoas infectadas pelo HIV (Gráfico 1). 
Gráfico 1 - Distribuição dos casos de HIV/Aids segundo sexo e ano de diagnóstico, Alagoas, 2009 a 2018.

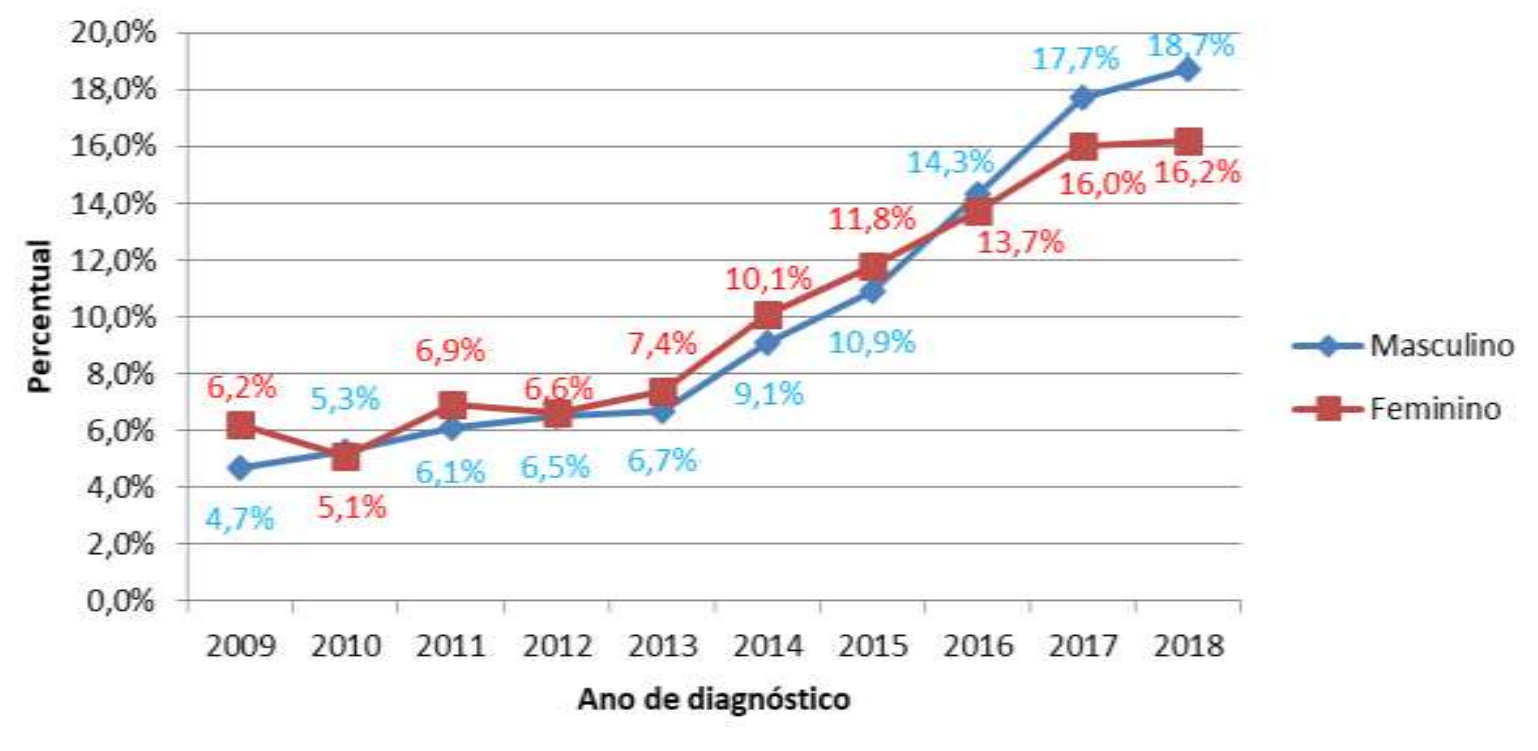

Fonte: SINAN (dados extraídos em 21/08/2019).

Outra tendência encontrada nos resultados do presente estudo é o processo de juvenização do sexo feminino, meninas com idade entre 15 e 19 anos (8,7\% das notificações entre as mulheres) apresentam mais que o dobro de percentual dos casos notificados quando comparadas ao sexo masculino de mesma faixa etária (4,0\%). O trabalho de Taquette (2010) mostra que no Brasil o processo de juvenização da Aids no sexo feminino ocorre na maioria das vezes por meio das relações sexuais desprotegidas, atrelada à vulnerabilidade social decorrente da pobreza e da violência de gênero, além da dificuldade de acesso aos serviços de saúde e da necessidade de adequação dessas meninas durante a adolescência à sociedade em que vivem.

Além disso, no que se refere às faixas etárias dos indivíduos, a análise mostra que a idade média dos diagnósticos de casos de HIV e Aids é de 35 anos, sendo os adultos jovens (idade que compreende entre os 20 e 40 anos) de ambos os sexos os mais afetados pela epidemia da Aids.

A expressividade desse dado também pode ser visualizada em âmbito nacional, onde pessoas em idade reprodutiva e laboral são as mais afetadas pela epidemia. No Brasil, entre os anos de 2007 e 2019, constatou-se que a maioria dos casos de infecção pelo HIV encontra-se na faixa de 20 a 34 anos, com percentual de 52,7\% dos casos. (Brasil, 2019a).

A disseminação da Aids em meio à faixa etária mais atingida de acordo com o presente estudo infelizmente revela uma problema social e psicológico para as pessoas vivendo com HIV (PVHIV) independente do sexo, uma vez que esses indivíduos além de conviverem com o vírus e com a possibilidade de desenvolver doenças oportunistas, convivem também com o estigma, o preconceito, medo da demissão no ambiente de trabalho, além do receio em perder relações sociais importantes, como entre amigos próximos e familiares (Botti et al., 2009; Freitas, 2012).

Os achados relacionados ao grau de escolaridade e à raça/cor dos indivíduos demonstram uma tendência de pauperização, acompanhando a tendência nacional onde a maioria dos casos de Aids se encontra entre as pessoas com baixos níveis de escolaridade e da raça/cor parda e preta (Brasil, 2019a).

$\mathrm{O}$ estado de Alagoas se encontra em uma das regiões mais pobres do país e enfrenta ao longo da sua história desigualdades sociais ligadas ao baixo índice de escolarização da população e ao baixo índice de renda (IBGE, 2019), e isso se reflete nos indivíduos afetados pelo HIV e pela Aids. Como pode ser observado no estudo, 44,5\% dos indivíduos notificados durante a série histórica são analfabetos ou possuem até o ensino fundamental completo, enquanto à raça/cor indicam que 5.325 indivíduos $(71,5 \%)$ são pardos, 821 indivíduos $(11,2 \%)$ são brancos e 629 indivíduos $(8,4 \%)$ são pretos. A raça/cor parda 
é predominante tanto nos homens quanto nas mulheres. Todavia, existe divergência quanto à segunda raça/cor de maior predominância entre os sexos. Em meio aos homens a segunda raça/cor predominante é branca com 580 indivíduos (12,4\%), enquanto que em meio às mulheres essa posição é ocupada pela raça/cor preta com 257 indivíduos $(9,3 \%)$.

No recorte temporal da pesquisa, o estado de Alagoas apresenta a via de transmissão sexual do HIV como a preponderante em número de casos notificados. Em seguida, aparece a via de transmissão parenteral e, por fim, a via de transmissão vertical (mãe para filho) em número de casos. Vale ressaltar a presença do alto percentual de casos com a informação sobre modo de transmissão ignorada (Tabela 1).

Tabela 1 - Distribuição dos casos de HIV/Aids segundo modo de transmissão, Alagoas, 2009 a 2018.

\begin{tabular}{ccc}
\hline Modo de transmissão & Frequência (n) & Porcentagem (\%) \\
\hline Vertical & 70 & $0,9 \%$ \\
Sexual & 5617 & $75,4 \%$ \\
Parenteral & 114 & $1,5 \%$ \\
Ignorado & 1646 & $22,1 \%$ \\
\hline Total & 7447 & $100,0 \%$ \\
\hline
\end{tabular}

Fonte: SINAN (dados extraídos em 21/08/2019).

É sabido que no Brasil e no mundo o modo de transmissão sexual se apresenta como a principal via de transmissão do HIV (Brasil, 2019a). Os resultados do presente trabalho seguem essa mesma linha, apontando que 75,4\% dos indivíduos infectaram-se através da via sexual.

Sob a ótica das categorias de exposição existentes na via de transmissão sexual Dourado et al. (2006) e Mombelli et al. (2015) afirmam que a categoria de exposição heterossexual é a mais comum para infecção do HIV/Aids para homens e mulheres. Tal afirmação pode ser constatada nos resultados obtidos por este estudo, onde se identifica que a categoria de exposição mais apontada nos casos notificados foi a categoria heterossexual, atingindo os maiores percentuais tanto entre os homens $(33,2 \%)$ quanto entre as mulheres $(83,5 \%)$.

No início da epidemia da Aids as relações homossexuais entre homens apresentavam grande relevância nos casos notificados de Aids, uma vez que esse grupo de indivíduos era um dos mais afetados pela infecção (Brasil, 2018a; Mombelli et al., 2015; Terto Jr., 2002). No presente estudo a categoria de exposição sexual formada por homens que têm relação sexual com outros homens apresenta um alto índice de transmissão do HIV/Aids através da relação homossexual (26,6\%) quando comparada a mulheres que também realizam práticas homossexuais $(0,7 \%)$.

É importante destacar que ao realizar-se uma busca acerca do assunto epidemiologia da Aids relacionada às questões da homossexualidade, percebe-se na literatura que o tema ainda é pouco difundido e bastante escasso, impossibilitando dessa forma uma melhor compreensão acerca de como a epidemia vem atingindo as várias populações de homens com práticas homossexuais e com quais outros problemas de saúde e doenças se cruzam (Terto Jr., 2002).

No que diz respeito ao modo de transmissão vertical o resultado aponta 70 indivíduos notificados, sendo que a maioria dos casos está representada por indivíduos do sexo masculino. Apesar dessa via de infecção não representar o principal modo de transmissão do HIV, ela é apontada em estudos científicos como uma das principais causas de infecção em crianças e responde por cerca de $80 \%$ dos casos de infecção no público infantil, o que sugere a existência de alguns casos observados dentro do estudo em questão (Carvalho et al., 2004; Friedrich, 2016). 
Ao longo da série histórica o modo de transmissão por via parenteral apresenta 1,53\% de todos os casos notificados. Em meio aos casos relativos a esse modo de transmissão, a categoria de exposição que mais chama a atenção no estudo é a composta por indivíduos que fazem uso de drogas injetáveis com 71 casos notificados $(62,3 \%)$.

Quanto ao uso de drogas injetáveis é importante salientar que, no período de 2009 a 2018, o banco de dados dos indicadores dos municípios brasileiros acerca do HIV/Aids aponta que o Brasil vem diminuindo o número de casos notificados de transmissão do HIV por meio do uso de drogas injetáveis, enquanto que no estado de Alagoas observa-se uma oscilação no número de casos ao longo dos anos (Brasil, 2019b).

No diagnóstico da infecção pelo HIV é possível que ocorram falhas e erros de diagnóstico. Segundo consta no Manual Técnico para o Diagnóstico da Infecção pelo HIV em Adultos e Crianças (2018b) elaborado pelo Ministério da Saúde, diversas podem ser as causas desses erros de diagnóstico, a saber: o período de janela diagnóstica; limitações dos testes ligados a sensibilidade e especificidade; equipamentos ou insumos mal armazenados e com falta de manutenção; adoção correta dos fluxogramas definidos no manual; e erros de cunho operacional como resultado com interpretação equivocada, realização errada do teste pelos profissionais habilitados e erros na identificação e contaminação cruzada entre as amostras.

O presente estudo temporal mostra que em Alagoas no período analisado houve o descarte de 29 casos notificados, ou seja, 29 casos de erros de diagnóstico. Esses erros de diagnóstico enquadram-se em uma das situações descritas no rol indicado no parágrafo anterior.

Quando observada a frequência relativa de cada um dos critérios de definição de casos (critério CDC Adaptado, critério Rio de Janeiro/Caracas, critério óbito, HIV+ e descartado) por ano de diagnóstico (tabela 2) é possível observar que o critério CDC Adaptado teve seus maiores percentuais registrados entre os anos de 2009 e 2013, apresentando um declínio significativo entre o ano de 2013 e o ano de 2014, reduzindo o percentual de 55,5\% para 31,2\%. O critério Rio de Janeiro/Caracas, por sua vez, apresentou seu maior percentual no ano de 2009 com 82 casos (21\%), enquanto que o critério óbito atingiu seu maior percentual em 2011 com 30 casos (6,3\%). Os casos registrados como HIV apresentam seus maiores percentuais entre os anos de 2014 e 2018. Já o descarte dos casos notificados apresentou um salto de 6 casos (0,5\%) registrados em 2017 para 14 casos $(1,1 \%)$ registrados em 2018, ano em que atingiu seu maior patamar em toda a série histórica analisada.

Tabela 2 - Distribuição dos casos de HIV/Aids segundo critério de definição de casos e ano de diagnóstico, Alagoas, 2009 a 2018.

\begin{tabular}{|c|c|c|c|c|c|c|c|c|c|c|c|c|}
\hline \multirow{3}{*}{$\begin{array}{c}\text { Ano de } \\
\text { diagnóstico }\end{array}$} & \multicolumn{12}{|c|}{ Critério de definição de caso } \\
\hline & \multicolumn{2}{|c|}{ CDC adaptado } & \multicolumn{2}{|c|}{$\begin{array}{c}\text { Rio de Janeiro / } \\
\text { Caracas }\end{array}$} & \multicolumn{2}{|c|}{ Óbito } & \multicolumn{2}{|c|}{$\mathrm{HIV}+$} & \multicolumn{2}{|c|}{ Descartado } & \multicolumn{2}{|c|}{ Total } \\
\hline & $\mathrm{n}$ & $\%$ & $\mathrm{n}$ & $\%$ & $\mathrm{n}$ & $\%$ & $\mathrm{n}$ & $\%$ & $\mathrm{n}$ & $\%$ & $\mathrm{~N}$ & $\%$ \\
\hline 2009 & 263 & $67,4 \%$ & 82 & $21,0 \%$ & 7 & $1,8 \%$ & 37 & $9,5 \%$ & 1 & $0,3 \%$ & 390 & $100,0 \%$ \\
\hline 2010 & 271 & $69,5 \%$ & 63 & $16,2 \%$ & 9 & $2,3 \%$ & 47 & $12,1 \%$ & 0 & $0,0 \%$ & 390 & $100,0 \%$ \\
\hline 2011 & 326 & $68,3 \%$ & 67 & $14,0 \%$ & 30 & $6,3 \%$ & 54 & $11,3 \%$ & 0 & $0,0 \%$ & 477 & $100,0 \%$ \\
\hline 2012 & 312 & $64,1 \%$ & 76 & $15,6 \%$ & 17 & $3,5 \%$ & 82 & $16,8 \%$ & 0 & $0,0 \%$ & 487 & $100,0 \%$ \\
\hline 2013 & 286 & $55,5 \%$ & 67 & $13,0 \%$ & 21 & $4,1 \%$ & 140 & $27,2 \%$ & 1 & $0,2 \%$ & 515 & $100,0 \%$ \\
\hline 2014 & 220 & $31,2 \%$ & 77 & $10,9 \%$ & 26 & $3,7 \%$ & 381 & $54,0 \%$ & 1 & $0,1 \%$ & 705 & $100,0 \%$ \\
\hline 2015 & 218 & $26,0 \%$ & 79 & $9,4 \%$ & 27 & $3,2 \%$ & 511 & $61,1 \%$ & 2 & $0,2 \%$ & 837 & $100,0 \%$ \\
\hline 2016 & 263 & $25,1 \%$ & 111 & $10,6 \%$ & 8 & $0,8 \%$ & 662 & $63,2 \%$ & 4 & $0,4 \%$ & 1048 & $100,0 \%$ \\
\hline 2017 & 342 & $26,9 \%$ & 79 & $6,2 \%$ & 15 & $1,2 \%$ & 831 & $65,3 \%$ & 6 & $0,5 \%$ & 1273 & $100,0 \%$ \\
\hline 2018 & 371 & $28,0 \%$ & 59 & $4,5 \%$ & 12 & $0,9 \%$ & 869 & $65,6 \%$ & 14 & $1,1 \%$ & 1325 & $100,0 \%$ \\
\hline Total & 2872 & $38,6 \%$ & 760 & $10,2 \%$ & 172 & $2,3 \%$ & 3614 & $48,5 \%$ & 29 & $0,4 \%$ & 7447 & $100,0 \%$ \\
\hline
\end{tabular}

Fonte: SINAN (dados extraídos em 21/08/2019). 
Os percentuais relativos ao critério HIV+ acentuam-se a partir do ano de 2014, reflexo da portaria $\mathrm{n}^{\mathrm{o}} 1.271 / 14$, que efetivou a notificação compulsória dos casos de HIV a partir do ano de 2014. Desta feita, o número de casos definidos apenas como HIV cresceu consideravelmente em âmbito nacional (Brasil, 2019a).

É válido destacar que o SINAN não constitui o sistema mais adequado para a análise do quantitativo de óbitos relacionados à Aids. Para tal finalidade existe o SIM, sistema específico em quantificar óbitos das mais diversas causas, incluindo a Aids (Medronho, 2009). Uma análise do SIM, contudo, não é parte constituinte dos objetivos da presente pesquisa.

O estudo permitiu ainda evidenciar a partir do ano de 2012 uma diminuição no número de óbitos em Alagoas, uma vez que os maiores percentuais de óbito por Aids correspondem aos anos de 2009 com 30,5\% dos casos e 2011 com 32,3\%. Esse dado permite fazer uma analogia com o que acontece no Brasil, que também vem apresentando resultados decrescentes no que diz respeito ao número de óbitos registrados por Aids (Brasil, 2019a).

Outro ponto a ser discutido refere-se à sobrevida dessas pessoas, que passou a aumentar ao longo dos anos, saltando de 66\% de indivíduos vivos em 2011 para 91,7\% em 2018, possivelmente em decorrência do uso da terapia antirretroviral que desde 1996 vem sendo distribuída no Brasil pelo SUS e permite que a carga viral das pessoas que tomam essa medicação chegue a níveis indetectáveis, aumentando dessa forma a sobrevida e diminuindo os sintomas causados pela Aids (Brasil, 2019c).

É válido enfatizar os estudos epidemiológicos realizados por Gonçalves (2008) e Pedrosa et al. (2015) que apontam que em estudos secundários apesar de haver algumas limitações no preenchimento dos registros ainda assim a análise não se compromete para fins de realização de alguns estudos epidemiológicos.

A incompletude de dados nos sistemas de informação da saúde é comum no Brasil, e este problema pode acarretar além da dificuldade de análises em estudos de cunho acadêmico, na impossibilidade do uso de variáveis relevantes para o planejamento, monitoramento e execução de políticas públicas de saúde (Romero \& Cunha, 2004; 2006; 2007).

No que se refere à apresentação de alguns dados epidemiológicos dispostos nos resultados do presente estudo de maneira não preenchida ou categorizados sob a denominação de ignorados, é válido ressaltar que a incompletude de tais dados não inviabilizou a análise, todavia quando os campos são preenchidos em sua integralidade a análise torna-se mais fidedigna para fins de estudos epidemiológicos.

\section{Considerações Finais}

O presente estudo aponta que a disseminação do HIV/Aids no estado de Alagoas ao longo da série histórica analisada - entre os anos de 2009 a 2018 - apresenta tendências de interiorização, juvenização, feminização e pauperização.

O estado de Alagoas apresenta aumento do número de novos casos notificados de HIV anualmente e diminuição do número de óbitos no período em análise, seguindo a mesma tendência observada na região nordeste do país.

O estudo revela ainda que ao longo dos anos em análise há uma diminuição na mortalidade das (PVHIV), o que denota uma melhoria na qualidade de vida dessas pessoas. Um processo colaborativo em meio a esse cenário positivo para as PVHIV são as políticas públicas do governo federal que desde 1996 vem distribuindo por meio do SUS medicamentos antirretrovirais para o tratamento de todas as pessoas infectadas ou expostas ao vírus HIV.

O governo a fim de melhorar a assistência médica às PVHIV e diminuir os dados referentes à infecção pelo vírus e o adoecimento da população que se encontra vulnerável e exposta ao HIV, vem ao longo dos anos criando campanhas publicitárias, distribuindo camisinhas, kits de Profilaxia Pré-Exposição (PrEP) e Profilaxia Pós-Exposição (PEP), além da elaboração de leis trabalhistas e de assistência que acolhem as pessoas acometidas pelo vírus.

Todavia, apesar dos esforços dos órgãos internacionais, do governo brasileiro, das entidades civis e dos órgãos não 
governamentais para o controle da epidemia da Aids, é sabido que essa situação se encontra ainda distanciada de seu fim, tendo em vista que não houve descoberta de cura até o presente momento.

A apresentação da doença nas diversas regiões brasileiras em forma de epidemia multifacetada requer a elaboração de políticas públicas específicas para cada região, o que enseja estudos mais bem elaborados e estruturados, que retratem de forma fidedigna o contexto epidemiológico de cada localidade, a exemplo do presente estudo que debruça sua análise acerca da realidade alagoana.

Observa-se como limitação do presente estudo os dados categorizados com o status de ignorado/não preenchido, que representam volumes consideráveis em algumas variáveis tornando a análise limitada. Tal incompletude de dados decorre de falha no momento de alimentação dos sistemas governamentais podendo ter causas diversas, a exemplo de carência de efetivo e/ou capacitação dos profissionais responsáveis por exercer atividades de cunho burocrático.

Sugere-se para trabalhos futuros um estudo acerca dos fatores que impactam diretamente no processo de incompletude de dados dos sistemas de informação de saúde, analisando suas implicações para a qualificação dos bancos de dados relativos aos sistemas de informação governamentais.

Aponta-se ainda como sugestão a investigação do processo de subnotificação dos casos de HIV e Aids nos municípios interioranos, tendo em vista que esse processo pode identificar outros municípios não listados, mas que apresentam casos, apontando possivelmente um processo de interiorização da epidemia ainda mais acentuado.

\section{Referências}

Botti, M. L., Waidman, M. A. P., Marcon, S. S., \& Scochi, M. J. (2009). Conflitos e sentimentos de mulheres portadoras de HIV/AIDS: um estudo bibliográfico. Revista da Escola de Enfermagem da USP, 43(1), 79-86. https://dx.doi.org/10.1590/S0080-62342009000100010

Brasil. Ministério da Saúde. Departamento de Doenças de Condições Crônicas e Infecções Sexualmente Transmissíveis. (2019a). Boletim Epidemiológico de HIV/Aids 2019. http://www.aids.gov.br/pt-br/pub/2019/boletim-epidemiologico-de-hivaids-2019.

Brasil. Ministério da Saúde. Departamento de Doenças de Condições Crônicas e Infecções Sexualmente Transmissíveis. (2018b). Manual Técnico para o Diagnóstico da Infecção pelo HIV em Adultos e Crianças. http://www.aids.gov.br/pt-br/node/57787.

Brasil. Ministério da Saúde. Departamento de Doenças de Condições Crônicas e Infecções Sexualmente Transmissíveis. Vigilância Epidemiológica. http://www.aids.gov.br/pt-br/gestores/vigilancia-epidemiologica\#_msocom_1.

Brasil. Ministério da Saúde. Secretaria de Vigilância em Saúde. Coordenação-Geral de Desenvolvimento da Epidemiologia em Serviços. (2019c). Guia de Vigilância em Saúde. Volume único. (3a ed.), 740 p. http://portalarquivos2.saude.gov.br/images/pdf/2019/junho/25/guia-vigilancia-saude-volume-unico3ed.pdf.

Brasil. Ministério da Saúde. Secretária de Vigilância em Saúde. Departamento de Doenças de Condições Crônicas e Infecções Sexualmente Transmissíveis. (2019b). Indicadores e dados básicos do HIV/Aids nos municípios brasileiros. http://indicadores.aids.gov.br/.

Brasil. Ministério da Saúde. Secretária de Vigilância em Saúde. Programa Nacional de DST e AIDS. (2018a). História da aids. http://www.aids.gov.br/ptbr/centrais-de-conteudos/historia-aids-linha-do-tempo.

Brito, A. M., Castilho, E. A., \& Szwarcwald, C. L. (2001). AIDS e infecção pelo HIV no Brasil: uma epidemia multifacetada. Revista da Sociedade Brasileira de Medicina Tropical, 34(2), 207-217. https://doi.org/10.1590/S0037-86822001000200010

Carvalho, R. L., Krahe, C., Farina, G., Paula, D. O., Richetti, N, \& Crossetti, T. (2004). Teste rápido para diagnóstico da infecção pelo HIV em parturientes. Revista Brasileira de Ginecologia e Obstetrícia, 26(4), 325-328. https://dx.doi.org/10.1590/S0100-72032004000400010

Dourado, I., Veras, M. A. S. M., Barreira, D., \& Brito, A. M. (2006). Tendências da epidemia de Aids no Brasil após a terapia anti-retroviral. Revista de Saúde Pública, 40, 9-17. https://doi.org/10.1590/S0034-89102006000800003

Fundação Oswaldo Cruz. Ministério da Saúde. O Vírus da Aids, 20 depois: A epidemia da aids através do tempo. http://www.ioc.fiocruz.br/aids20anos/linhadotempo.html

Freitas, J. G., Galvão, M. T. G., Araujo, M. F. M., Costa, Ê., \& Lima, I. C. V. (2012). Enfrentamentos experienciados por homens que vivem com HIV/Aids no ambiente de trabalho. Revista da Escola de Enfermagem da USP, 46(3), 720-726. https://dx.doi.org/10.1590/S0080-62342012000300026

Friedrich, L., Menegotto, M., Magdaleno, A. M., \& Silva, C. L. O. (2016). Transmissão vertical do HIV: uma revisão sobre o tema. Boletim Científico de Pediatria, 05(3), 81-86. http://www.sprs.com.br/sprs2013/bancoimg/170118174005bcped_05_03_a03.pdf

Gonçalves, V. F., Kerr, L. R. F. S., Mota, R. M. S., Mota, J. M. A. (2008). Estimativa de subnotificação de casos de AIDS em uma capital do Nordeste. Revista Brasileira de Epidemiologia. 11(3), 356-364. https://www.scielosp.org/pdf/rbepid/2008.v11n3/356-364. 
Research, Society and Development, v. 10, n. 5, e15810514811, 2021

(CC BY 4.0) | ISSN 2525-3409 | DOI: http://dx.doi.org/10.33448/rsd-v10i5.14811

Instituto Brasileiro de Geografia e Estatística. Diretoria de Pesquisas, Coordenação de População e Indicadores Sociais, Estimativas da população residente com data de referência $1^{\circ}$ de julho de 2019. (2019). Cidades e Estados: Alagoas. https://www.ibge.gov.br/cidades-e-estados/al.html.

Mombelli, M. A., Barreto, M. S., Arruda, G. O., \& Marcon, S. S. (2015). Epidemia da aids em tríplice fronteira: subsídios para a atuação profissional. Revista Brasileira de Enfermagem, 68(3), 429-437. https://dx.doi.org/10.1590/0034-7167.2015680308i

Medronho, R. A., Bloch, K. V., Luiz, R. R., Werneck, G. L. (2009). Epidemiologia (2a ed.). Atheneu.

Paiva, M. S. (1999). A feminização da aids: uma questão de gênero? Revista Brasileira de Enfermagem. 52:7-13. https://www.scielo.br/pdf/reben/v52n1/v52n1a02.pdf.

Pedrosa, N. L., Paiva, S. S., Almeida, R. L. F, Holanda, E. R., Kerr, L. R. F. S., \& Galvão, M. T. G. (2015). Série histórica da AIDS no Estado do Ceará, Brasil. Ciência \& Saúde Coletiva, 20(4), 1177-1184. https://doi.org/10.1590/1413-81232015204.00582014

Prodanov, C. C., Freitas, E. C. (2013). Metodologia do trabalho científico: métodos e técnicas da pesquisa e do trabalho acadêmico (2a ed.). Novo Hamburgo: Feevale.

Reis, C. T., Czeresnia, D., Barcellos, C., \& Tassinari, W. S. (2008). A interiorização da epidemia de HIV/AIDS e o fluxo intermunicipal de internação hospitalar na Zona da Mata, Minas Gerais, Brasil: uma análise espacial. Cadernos de Saúde Pública, 24(6), 1219-1228. https://doi.org/10.1590/S0102$311 X 2008000600003$

Romero, D. E., \& Cunha, C. B. (2007). Avaliação da qualidade das variáveis epidemiológicas e demográficas do Sistema de Informações sobre Nascidos Vivos, (2002). Cadernos de Saúde Pública, 23(3), 701-714. https://dx.doi.org/10.1590/S0102-311X2007000300028

Romero, D. E. R. \& Cunha, C. B. (2004). Avaliação do grau de preenchimento e definições das variáveis sociais no Sistema de Informação de Nascidos Vivos Diferenciais por Unidade Federada. Anais do XIV Encontro Nacional de Estudos Populacionais. ABEP - Associação brasileira de estudos populacionais. http://www.abep.org.br/publicacoes/index.php/anais/issue/view/34

Romero, D. E. R. \& Cunha, C. B. (2006). Disponibilidade e qualidade da informação epidemiológica e demográfica no Sistema de Informações sobre Nascidos Vivos (SINASC) e no Sistema de Informação de Mortalidade (SIM) para menores de um ano. Anais do XIV Encontro Nacional de Estudos Populacionais. ABEP - Associação brasileira de estudos populacionais. http://www.abep.org.br/publicacoes/index.php/anais/article/view/1545

Sidra - Sistema IBGE de Recuperação Automática. Estimativas de População Tabela 6579 - População residente estimada. https://sidra.ibge.gov.br/tabela/6579\#resultado

Taquette, S. R. (2010). Interseccionalidade de gênero, classe e raça e vulnerabilidade de adolescentes negras às DST/aids. Saúde e Sociedade, 19(2), 5162. https://doi.org/10.1590/S0104-12902010000600006

Terto Jr., V. (2002). Homossexualidade e saúde: desafios para a terceira década de epidemia de HIV/AIDS. Horizontes Antropológicos, 8(17), 147158. https://dx.doi.org/10.1590/S0104-71832002000100008

Unaids - Programa Conjunto das Nações Unidas sobre HIV/AIDS. (2012). A ONU e a resposta à aids no Brasil. Geneva: UNAIDS. https://unaids.org.br/wpcontent/uploads/2016/03/A-ONU-e-a-resposta-PORTUGU\%C3\%8AS.pdf. 